\title{
Long-Gap Esophageal Atresia Is a Unique Entity within the Esophageal Atresia Defect Spectrum
}

\author{
Sigrid Bairdain ${ }^{a}$ David Zurakowski ${ }^{a}, b$ Sara O. Vargas ${ }^{c}$ Nicole Stenquist $^{b}$ \\ Molly McDonald ${ }^{b}$ Meghan C. Towne ${ }^{d}$ David T. Miller $^{e} f \quad$ Russell W. Jennings ${ }^{a}$ \\ David B. Kantor ${ }^{\text {b }}$ Pankaj B. Agrawald,e,g \\ ${ }^{a}$ Department of Pediatric Surgery, ${ }^{b}$ Division of Critical Care Medicine, Department of Anesthesiology, Perioperative \\ and Pain Medicine, ${ }^{\mathrm{C}}$ Department of Pathology, ${ }^{\mathrm{d}}$ Gene Discovery Core, Manton Center for Orphan Disease Research, \\ e Division of Genetics and Genomics, ${ }^{f}$ Claritas Genomics, and ${ }^{9}$ Division of Newborn Medicine, Department of \\ Medicine, Boston Children's Hospital, Harvard Medical School, Boston, Mass., USA
}

\section{Key Words}

Esophageal atresia - Long-gap esophageal atresia ·

VACTERL $\cdot$ Chromosomal microarray analysis

\begin{abstract}
Background: Long-gap esophageal atresia (LGEA) may have clinical and syndromic presentations different from those of esophageal atresia (EA) that affects shorter segments of the esophagus (non-LGEA). This may suggest unique underlying developmental mechanisms. Objectives: We sought to characterize clinical differences between LGEA and non-LGEA by carefully phenotyping a cohort of EA patients, and furthermore to assess molecular genetic findings in a subset of them. Methods: This is a retrospective cohort study to systematically evaluate clinical and genetic findings in EA infants who presented at our institution over a period of 10 years (2005-2015). Results: Two hundred twenty-nine EA patients were identified, 69 (30\%) of whom had LGEA. Tracheoesophageal fistula was present in most non-LGEA patients (158 of 160) but in only $30 \%$ of LGEA patients. The VACTERL association was more commonly seen with nonLGEA compared to LGEA (70 vs. $25 \%$; $p<0.001$ ). Further, tri-
\end{abstract}

\section{KARGER}

(c) 2016 S. Karger AG, Basel

E-Mail karger@karger.com

www.karger.com/neo somy 21 was more common in LGEA than in non-LGEA. 25\% of LGEA patients had an isolated EA diagnosis without other anomalies, compared to $<1 \%$ for non-LGEA. Chromosomal microarray analysis showed copy number variations (CNV) in 4 of 39 non-LGEA patients and 0 of 3 LGEA patients. A review of the ClinGen database showed that none of those CNV have been previously described with EA. Conclusions: LGEA represents a unique type of EA. Compared to non-LGEA, it is more likely to be an isolated defect and associated with trisomy 21. Further, it is less commonly seen with VACTERL anomalies. Our findings suggest the involvement of unique pathways that may be distinct from those causing non-LGEA.

(c) 2016 S. Karger AG, Basel

\section{Introduction}

Esophageal atresia (EA) is a congenital defect wherein the upper esophagus ends in a blind pouch and is not connected to the lower esophagus. EA is often associated with a tracheoesophageal fistula (TEF) that represents an abnormal opening between the trachea and the esophagus [1]. There are several classification schemas for EA, one 
of which divides EA into long-gap EA (LGEA) and nonLGEA, critical in the surgical repair approach. LGEA is defined by a gap length $>3 \mathrm{~cm}$ between the upper and lower esophageal segments or more than 2 vertebral bodies making the gap too big to repair by primary anastomosis [1-6]. This distance ultimately delineates the timing and ease of repair. LGEA does not have a distal TEF, and it may or may not have a proximal TEF [1-6]. LGEA is associated with a high morbidity, and there is a lack of consensus regarding its surgical management. Foker et al. [2] described the utilization of external traction sutures to promote in vivo growth through tension-induced lengthening, and subsequent delayed primary repair, potentially avoiding the need for interpositions $[2,3]$.

The developmental events leading to EA are poorly understood and may involve multifactorial gene-environment interactions with heterogeneous genetic contributions. Single gene mutations have been identified only in rare cases, and the exact genetic etiology remains largely unknown [7]. We reasoned that classifying EA patients into similar phenotypic categories might improve the likelihood of identifying the biological events leading to aberrant organogenesis. Given that our institution is a center that encounters a high volume of LGEA patients, we sought to ascertain the differences between LGEA and non-LGEA patients, in particular differences between rates of associated clinical/phenotypic conditions and syndromes and molecular genetic aberrations.

\section{Materials and Methods}

\section{Patient Enrollment and Data Collection}

This study was approved by the Boston Children's Hospital's (BCH) Institutional Review Board. We reviewed medical information on all EA patients managed and followed at our institution from 2005 through 2015 via a collaborative effort between the $\mathrm{BCH}$-based Gene Discovery Core of the Manton Center for Orphan Disease Research and the EA Multidisciplinary Treatment Team. Patients were evaluated in both inpatient and outpatient settings.

Clinical data collected included sex and primary diagnosis, as well as the presence of esophageal, cardiac, renal, limb, spinal, anorectal, or other malformations. For the purpose of the current study, EA patients were considered to have LGEA when a primary anastomosis was not possible due to the length of the gap between the upper and lower esophageal segments as described earlier [16]. Disorders of the esophagus were further classified as follows: (1) LGEA without a TEF, (2) LGEA with a proximal TEF, (3) nonLGEA with a distal TEF or type C EA, (4) non-LGEA without a TEF, and (5) TEF without EA (H-type TEF). Anomalies that specifically occur within VACTERL were recorded; those who had at least 3 of those anomalies were characterized as having VACTERL. Other anomalies and syndromic associations were also recorded.
Table 1. Classification of EA and its relationship with TEF

\begin{tabular}{lc}
\hline Type of EA & $\mathrm{n}(\%)$ \\
\hline Non-LGEA with a distal TEF (type C EA) & $153(67)$ \\
LGEA without a TEF & $48(21.0)$ \\
LGEA with a proximal TEF & $21(9.2)$ \\
H-type TEF without EA & $5(2.2)$ \\
Non-LGEA without a TEF & $1(0.4)$ \\
Other $^{\mathrm{a}}$ & $1(0.4)$ \\
\hline
\end{tabular}

Total $\mathrm{n}=229$. ${ }^{\mathrm{a}}$ Squamous cell carcinoma of the esophagus by biopsy.

\section{Chromosomal Microarray Analysis}

During the study period, an in-house chromosomal microarray analysis (CMA) was performed as follows: whole genome oligonucleotide array comparative genomic hybridization arrays (Agilent 244K G4411B; Agilent Technologies, Palo Alto, Calif., USA) were used to detect copy number variations (CNV). Beginning in 2011, samples were tested with a custom Agilent 4x180K array designed for whole genome coverage. Both arrays were able to detect CNV greater than 35-50 kb throughout the genome. Images were captured by an Agilent scanner and quantified using Feature Extraction software (v9.0). CGH analytic software (v3.4) was subsequently used for data normalization, quality evaluation, and data visualization. Copy number aberrations were identified using the ADM-2 (aberration detection method 2) algorithm. For patients without in-house CMA testing, records from outside testing were procured when available. Detected $\mathrm{CNV}$ were analyzed in the ClinGen database to determine any associations with foregut malformations.

\section{Statistical Analysis}

Comparisons of proportions were made regarding the incidence of VACTERL between the LGEA and non-LGEA groups, as well as specific phenotypes, using Fisher's exact test. The distributions of the number of phenotypes between LGEA and non-LGEA groups were compared using Pearson's $\chi^{2}$ test. Statistical analysis was performed using the IBM/SPSS software package (version 22.0; IBM, Armonk, N.Y., USA). Two-tailed $p$ values $<0.05$ were considered statistically significant. The sample sizes of 69 LGEA and 160 non-LGEA patients provided $80 \%$ power to detect effect sizes based on $20 \%$ group differences in the percentage with VACTERL and each phenotype using a 2-group Fisher's exact test (nQuery Advisor version 7.0; Statistical Solutions, Cork, Ireland) [8, 9].

\section{Results}

During the study period, 229 patients were identified to have EA. Sixty-nine (30\%) had LGEA, while the remaining 160 were classified as non-LGEA (70\%). Fiftythree percent of all EA patients were males. The relationship between TEF and EA is summarized in table 1. One 
Table 2. Cardiac anomalies between LGEA and non-LGEA patients

\begin{tabular}{lcl}
\hline Cardiac subtype & $\begin{array}{l}\text { LGEA } \\
(\mathrm{n}=69)\end{array}$ & $\begin{array}{l}\text { Non-LGEA } \\
(\mathrm{n}=160)^{\mathrm{a}}\end{array}$ \\
\hline No cardiac abnormality & $39(57)$ & $50(32)$ \\
Septal defect (VSD or ASD) & $11(16)$ & $32(20)$ \\
Arch anomaly & $5(7)$ & $16(10)$ \\
Septal defect + arch anomaly & $10(15)$ & $29(19)$ \\
Tetralogy of Fallot & $2(3)$ & $16(10)$ \\
Otherc & $2(3)$ & $14(9)$ \\
\hline
\end{tabular}

Values are presented as numbers (\%). VSD $=$ Ventricular septal defect; ASD = atrial septal defect. ${ }^{\text {a }}$ Three patients had no cardiac documentation. ${ }^{\mathrm{b}}$ Includes abnormalities of the aortic arch and/or vessels entering into it. ${ }^{\mathrm{c}}$ Includes hypoplastic left heart syndrome and valvular stenosis.

hundred fifty-three (67\%) individuals had non-LGEA with a distal TEF (type C EA), while 48 (21\%) had LGEA without a TEF, 21 (9\%) had LGEA with a proximal TEF, 5 (2\%) had an H-type TEF without EA, and $1(0.4 \%)$ had non-LGEA without a TEF (table 1 ).

Of the entire EA cohort, 129 (56\%) patients met the criteria for VACTERL association. The VACTERL association was significantly more common in non-LGEA patients than in those with LGEA (112 or 70 vs. 17 or $25 \%$; $\mathrm{p}<0.001)$. In those 129 patients with VACTERL, the number of associated anomalies ranged from 3 to 7 (with 7 being the full complement of all associated anomalies). TEF was the most common (occurring in $94 \%$ of EA + VACTERL patients), closely followed by cardiac (in 78\%), vertebral (in 63\%), and renal (in 62\%) defects.

Patients with LGEA were more likely than those with non-LGEA to have isolated EA with no other anomalies ( 25 vs. $1 \%$; $\mathrm{p}<0.0001$ ). The most common anomalies seen in LGEA patients were cardiac (44\%), TEF (30\%), and renal and vertebral (25\% each). In contrast, the anomalies often present in non-LGEA patients were TEF (99\%) and cardiac (68\%) and vertebral (45\%) defects (fig. 1). The presence of VACTERL defects (vertebral, anal, cardiac, tracheoesophageal, renal, and limb defects that include poorly developed or missing thumbs or underdeveloped forearms and hands) were statistically more common in the non-LGEA group compared to the LGEA group (fig. 1). Non-VACTERL anomalies including other limb defects (leg length discrepancy, tibial abnormality, and femoral anteversion), conus defects (tethered cord and fatty filum), gastrointestinal anomalies (duodenal atresia, malrotation, and annular pancreas), facial defects

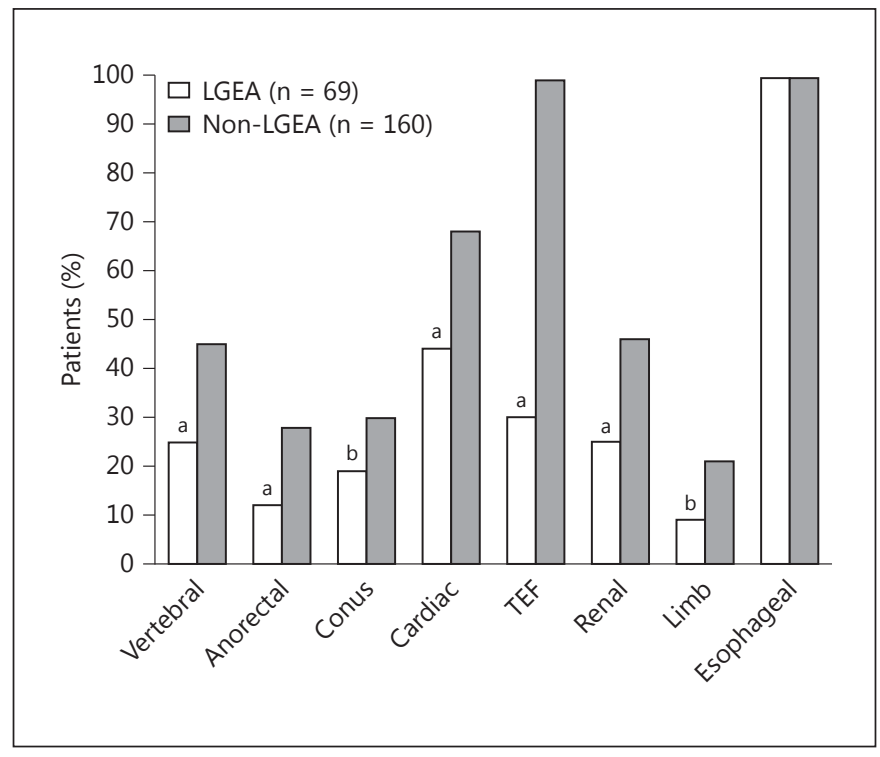

Fig. 1. Distribution of phenotypic categories among LGEA and non-LGEA patients. Comparisons include vertebral, anorectal, conus, cardiac, TEF, renal, limb, and esophageal defects. ${ }^{a}$ Significant group differences based on Fisher's exact test at the $\mathrm{p}<0.01$ level. ${ }^{\mathrm{b}}$ Differences between the 2 groups at $\mathrm{p}<0.05$.

(cleft lip and/or palate), and hydrocephalus were seen in both LGEA and non-LGEA patients. Conus and nonVACTERL limb defects were present more often in nonLGEA patients. Examining the types of cardiac defects more closely, we did not find a difference in the frequency of a concurrent diagnosis of aortic arch anomaly or septal defects in LGEA patients compared to non-LGEA patients (table 2).

EA occurred as part of a known genetic or chromosomal syndrome in 22 patients. The presence of an associated known genetic or chromosomal syndrome was significantly higher in LGEA patients than in non-LGEA patients ( $20 \mathrm{vs.} 5 \%$; $=0.001$ ). Trisomy 21 was a common chromosomal defect in LGEA patients $(9 / 69$, vs. $1 / 160$ in non-LGEA patients; $\mathrm{p}<0.0001)$. In contrast, 2 cases each of CHARGE syndrome due to the CHD7 mutation, and trisomy 18 , were only seen in non-LGEA patients.

In addition to investigating EA as part of known genetic and chromosomal syndromes, we also examined CMA data to identify any associations between EA and CNV. Of the 42 CMA test results available from the entire cohort, only 4 were noted to have a CNV. All of the detected CNV occurred in non-LGEA patients and were of parental origin. No available evidence demonstrates an association between the $\mathrm{CNV}$ observed here and foregut malformations. 


\section{Discussion}

In the largest retrospective cohort study yet of phenotypic variation in patients with LGEA, we stratified patients with EA according to the presence or absence of LGEA and then characterized these patients for associated congenital anomalies, syndromes, and CNV. Our goal was to determine whether the LGEA and non-LGEA phenotypes had similar presentations (except for the gap length that defines them), which would suggest a common defect in developmental programming. We observed that, compared to non-LGEA cases, LGEA cases are less likely to have a TEF and present as a VACTERL association, and they are more likely to occur as an isolated anatomical abnormality and have trisomy 21 . To further test whether this finding is unique to LGEA, we divided our cohort into patients with and without TEF and asked whether there were differences in the associated anomalies. In contrast to the case with LGEA, we found no significant differences between TEF and nonTEF patients in any of the VACTERL anomalies (vertebral, $p=0.05$; anorectal, $p=0.364$; cardiac, $p=0.140$; re$\mathrm{nal}, \mathrm{p}=0.267$, and limb, $\mathrm{p}=0.753$ ). These findings suggest the possibility that that LGEA represents an abnormality of developmental events or timing that may be distinct from non-LGEA, and it is not merely a severe manifestation of EA. This interpretation is broadly consistent with available data concerning the genetic and environmental factors that might contribute to esophageal malformations [10].

How can we reconcile our results with a previous study [11] that found no statistically difference in the occurrence VACTERL-associated anomalies between subjects with LGEA and non-LGEA? It is notable that the rate of detection of VACTERL anomalies was considerably higher in our study, raising the possibility that we detected these anomalies with a greater sensitivity. A second possibility is that non-LGEA patients with higher numbers of VACTERL anomalies are preferentially referred to our hospital.

The genetic basis of EA remains poorly understood, with only in a small fraction of patients carrying chromosomal defects or single gene defects [1,12-16]. We evaluated the role of CMA in EA infants with an unknown etiology (i.e. those patients without a known genetic or chromosomal diagnosis) and identified $4 \mathrm{CNV}$ among the 42 patients analyzed. Reviewing the ClinGen database, we found that one of these CNV was associated with abnormal development $[17,18]$, but it was not associated with EA or other foregut malformations. As such, in our cohort, CMA analysis did not provide any additional insight into the etiology of EA. It is important to note that CMA is not able to resolve smaller deletions and duplications, for example at the level of individual genes, which could potentially be revealed by other analytic methods such as Sanger sequencing.

There are a few limitations of our study. It is a retrospective single-institution study, and some of the findings may not be generalizable. For example, compared to other studies, we had a high proportion of LGEA patients, which is attributable to our institution being a referral center for LGEA [11]. Our cohort included complex patients, often transferred from other facilities, with complicated records and potentially inconsistent data collection, raising the possibility that despite all efforts some anatomical anomalies may have been missed. In addition, our CMA analysis covered only a subset of patients; this costly testing had been used clinically in only a subset of patients, and our study funds did not cover researchbased testing for this cohort.

In summary, in this study, we characterized a large cohort of subjects with EA and identified LGEA as a discrete phenotypic category that may reflect distinct developmental or potentially genetic etiologies. Genetic studies using various approaches including whole genome sequencing and identification of somatic mutations focused on this unique EA subgroup may help understand the underlying molecular defects.

\section{Acknowledgements}

This work was made possible by grants NIH T32 HD040128 and NIH K12 HD047349 (D.B.K.) and NIH/NIAMS 1R01AR068429-01 and NICHD/NHGRI/NIH U19HD077671 (P.B.A.). The authors would like to thank the many families who volunteered for this research study and the research personnel of the Manton Center and the EA Team for their continued dedication to this project.

\section{Disclosure Statement}

D.T.M. is part-time clinical consultant and medical director for Claritas Genomics, Inc., a majority-owned subsidiary of the $\mathrm{BCH}$ (nonequity professional service agreement). The remaining authors have no potential conflicts of interests to disclose. 


\section{References}

1 Spitz L: Oesophageal atresia. Orphanet J Rare Dis 2007;2:24.

2 Foker JE, Linden BC, Boyle EM, Marquardt C: Development of a true primary repair for the full spectrum of esophageal atresia. Ann Surg 1997;226:533-541.

3 Bairdain S, Kelly DP, Tan C, Dodson B, Zurakowski D, Jennings RW, et al: High incidence of catheter-associated venous thromboembolic events in patients with long gap esophageal atresia treated with the Foker process. J Pediatr Surg 2014;49:370-373.

4 Gross RE: The Surgery of Infancy and Childhood. Philadelphia, Saunders, 1953.

5 David TJ, O'Callaghan SE: Oesophageal atresia in the South West of England. J Med Genet 1975;12:1-11.

6 Al-Shanafey S, Harvey J: Long gap esophageal atresia: an Australian experience. J Pediatr Surg 2008;43:597-601.

7 Reutter H, Ludwig M: VATER/VACTERL association: evidence for the role of genetic factors. Mol Syndromol 2013;4:16-19.

8 Rosner B: Fundamentals of Biostatistics, ed 6. Belmont, Thomson Brooks/Cole, 2006, pp 184-193.

9 Vittinghoff E, Glidden DV, Shiboski SC, McCulloch CE: Regression Methods in Biostatistics: Linear, Logistic, Survival, and Repeated Measures Models. New York, Springer, 2005, pp 266-289.
10 Felix JF, de Jong EM, Torfs CP, de Klein A, Rottier RJ, Tibboel D: Genetic and environmental factors in the etiology of esophageal atresia and/or tracheoesophageal fistula: an overview of the current concepts. Birth Defects Res A Clin Mol Teratol 2009;85:747754.

11 Aslanabadi S, Ghabili K, Rouzrokh M, Hosseini MB, Jamshidi M, Adl FH, Shoja MM: Associated congenital anomalies between neonates with short-gap and long-gap esophageal atresia: a comparative study. Int J Gen Med 2011;4:487-491.

12 La Placa S, Giuffrè M, Gangemi A, Di Noto S, Matina F, Nociforo F, et al: Esophageal atresia in newborns: a wide spectrum from the isolated forms to a full VACTERL phenotype? Ital J Pediatr 2013;39:45.

13 Quan L, Smith DW: The VATER association: vertebral defects, anal atresia, T-E fistula with esophageal atresia, radial and renal dysplasia - a spectrum of associated defects. J Pediatr 1973;82:104-107.
14 Brosens E, Eussen H, van Bever Y, van der Helm RM, Ijsselstijn H, Zaveri HP, et al: VACTERL association etiology: the Impact of de novo and rare copy number variations. Mol Syndromol 2013;4:20-26.

15 Bartels E, Jenetzky E, Solomon BD, Ludwig M, Schmiedeke E, Grasshoff-Derr S, et al: Inheritance of the VATER/VACTERL association. Pediatr Surg Int 2012;28:681-685.

16 Choinitzki V, Zwink N, Bartels E, Baudisch F, Boemers TM, Hölscher A, et al: Second study on the recurrence risk of isolated esophageal atresia with or without trachea-esophageal fistula among first-degree relatives: no evidence for increased risk of recurrence of EA/ TEF or for malformations of the VATER/ VACTERL association spectrum. Birth Defects Res A Clin Mol Teratol 2013;97:786791.

17 ClinGen CNV nssv577415. https://genome. ucsc.edu/cgi-bin/hgc?hgsid=427207385_j4p 3I4OS4avJo35UR279CtoDWmH3\&c=chr13 $\& o=20823292 \& \mathrm{t}=21095898 \& \mathrm{~g}=\mathrm{iscaPathoge}$ nic\&i=nssv577415 (accessed May 19, 2015).

18 ClinGen CNV nssv577416. https://genome. ucsc.edu/cgi-bin/hgc?hgsid=427207385_j4p 3I4OS4avJo35UR279CtoDWmH3\&c=chr13 $\& o=20823292 \& \mathrm{t}=21095898 \& \mathrm{gg}=\mathrm{iscaPathoge}$ nic\&i=nssv577416 (accessed May 19, 2015). 